A. Morrison, J.J. Leitch, G. Szymanski ,G.Moula, B.Barlow, I.J.Burgess, B.Shobeir, H. Huang, J. Lipkowski, "Mechanism of Electrochemical Dissolution of Nickel Grown by Carbonyl Method", Electrochim Acta, 2017,248,112-122.

\title{
Mechanism of Electrochemical Dissolution of Nickel Grown by Carbonyl Method
}

\author{
A. Morrison ${ }^{1}$, J. J. Leitch ${ }^{1}$, G. Szymanski ${ }^{1}$,G. Moula ${ }^{1}$, B. Barlow ${ }^{2}$, I. J. Burgess ${ }^{2}$, B. Shobeir ${ }^{3}$,
}

\section{H. Huang ${ }^{3}$, J. Lipkowski ${ }^{1, *}$}

${ }^{1}$ Department of Chemistry, University of Guelph, 50 Stone Road East, Guelph, ON, Canada, N1G 2W1

${ }^{2}$ Departement of Chemistry, University of Saskatchewan, Saskatoon, SK, Canada, S7N 5C9

${ }^{3}$ Vale Base Metals Technology Development, 2060 Flavelle Boulevard, Sheridan Park, Mississauga, ON, Canada L5K 1Z9; E-mail address

*Corresponding author: jlipknow @uoguelph.ca

\begin{abstract}
Laboratory grown nickel sample was used to investigate the galvanostatic dissolution under industrial electroplating conditions. The sample contained multiple layers corresponding to changes in the $\mathrm{Ni}(\mathrm{CO})_{4}$ decomposition temperature ranging from 200 to $280^{\circ} \mathrm{C}$. Cyclic voltammetry curves were used to determine the active dissolution, passive and transpassive potentials regions of the sample. Chronopotentiometric curves recorded during galvanostatic dissolution displayed oscillations indicating that the dissolution mechanism involved the breakdown of the passive layer and repassivation of the surface. Scanning electron microscopy
\end{abstract}


(SEM) and white light interference microscopy (WLIM) were used to observe changes in morphology and roughness of the surface. The SEM images revealed the formation of surface pits with lacy covers at the surface during initial stages of the dissolution. At increased dissolution times, a transition from the lacy covered pits to open pits was observed. The images showed preferential dissolution occurring on $\mathrm{Ni}$ layers grown at the higher $\mathrm{Ni}(\mathrm{CO})_{4}$ decomposition temperatures. At longer dissolution times the preferential dissolution is also taking place at the boundaries between $\mathrm{Ni}$ laminas corresponding to $\mathrm{Ni}(\mathrm{CO})_{4}$ decomposition at different temperatures. .

\section{Introduction}

Electrolytic and carbonyl method purified nickel are the two forms of $\mathrm{Ni}$ substrates used in electroplating industry to plate commercial products. The carbonyl method, discovered by Ludwig Mond [1,2], involves passing CO over solid impure nickel at a moderate temperatures of $50-60^{\circ} \mathrm{C}$ to create gaseous carbonyl nickel, $\mathrm{Ni}(\mathrm{CO})_{4}$. The carbonyl nickel gas is then decomposed to form pure $\mathrm{Ni}_{(\mathrm{s})}$ pellets by heating it to temperatures $\left(200-280^{\circ} \mathrm{C}\right)$. The overall reaction is shown below:

$\mathrm{Ni}_{(s)}+4 \mathrm{CO}_{(\mathrm{g})} \stackrel{50^{\circ} \mathrm{C}-60^{\circ} \mathrm{C}}{\longrightarrow} \mathrm{Ni}(\mathrm{CO})_{4(g)} \stackrel{200^{\circ} \mathrm{C}-280^{\circ} \mathrm{C}}{\longrightarrow} \mathrm{Ni} i_{(s)_{\text {pure }}}+4 \mathrm{CO}_{(g)}$

The industrial Mond process proceeds as follows; first "seed pellets" $(\sim 1 \mathrm{~mm}$ in diameter) are pre-heated to the desired decomposition temperature and transferred to a chamber with $\mathrm{Ni}(\mathrm{CO})_{4}$. The gaseous carbonyl nickel decomposes and plates the pellets surface with a pure nickel film. This process is repeated about 300 times to grow the pellets layer by layer until an optimal diameter of $10 \mathrm{~mm}$ is reached [3,4]. The finished pellets have onion-like lamellar structures. Since the decomposition temperature may vary under industrial conditions, the 
properties of each lamina may also vary and these changes could affect the anodic dissolution behavior of the pellets during electroplating. Therefore, it is important to understand how the variations in the decomposition temperature affect the anodic dissolution of Ni pellets. The aim of this project is to investigate the dissolution of a laboratory grown $\mathrm{Ni}$ sample where the decomposition temperature of each lamina was varied in a controlled fashion. In this manner, we are able to evaluate the effect of variable decomposition temperature on the anodic $\mathrm{Ni}$ dissolution. In addition, this study contributes to the understanding of pit formation. We will show real time optical microscopy images, illustrating the dynamics of pit formation. We will demonstrate that during $\mathrm{Ni}$ dissolution, open pit formations are preceded by formation of pits with lacy covers. Electrochemical methods will be used to study the dissolution mechanism of the carbonyl Ni and White Light Interference Microscopy (WLIM) will be used to observe the evolution of the surface morphology over the duration of the dissolution. Additionally, Scanning Electron Microscopy (SEM) implemented with an Energy Dispersive Spectroscopy (EDS) detector will be used to analyze the texture and grain distribution, during the progression of the dissolution.

\section{Experimental}

A laboratory grown carbonyl purified nickel sample (denoted CN1) was obtained from Vale Canada (Mississauga, ON, CA). The CN1 sample consisted of five Ni laminae (each 200 $\mu \mathrm{m}$ thick) formed at decomposition temperatures $200,220,240,260$ and $280^{\circ} \mathrm{C}$, respectively. A titanium wire was welded to the sample, to provide electrical contact during the dissolution experiment. In order to observe selective dissolution along one face of the CN1 sample, the pellet was encased in an epoxy resin. The sample was cut in the direction perpendicular to the lamellar surface and then polished to a mirror-like finish to observe the initial grain structure. 
The LECO Vari/Pol system (Type: VP-50, LECO Corp., St. Joseph, MI, USA) was used to manually polish the sample gradually using finer grit polishing paper series $(240,320,400,600$ and 1200 grit). Using a polycrystalline diamond polishing suspension (Pace Technologies, Tucson, AZ, USA) of different sizes (15, 6, 3, and $1 \mu \mathrm{m}$ ) and polishing pads (Pace Technologies, Tucson, AZ, USA), the fine polishing was completed. This step is the most important in the polishing procedure in order to remove any defects and scratches which would interfere with imaging of the pristine grain structure. The final step is the ultra-fine polishing using a silicon suspension $(0.05 \mu \mathrm{m})$ along with an ultra-fine polishing pad on a vibro polisher (Type: GIGA0900, Pace Technologies, Tucson, AZ, USA). This method gives a mirror finish with a surface roughness $<0.05 \mu \mathrm{m}$ allowing for precise grain structure imaging. In order to remove any residual polishing material remaining after washing, a $4 \% \mathrm{H}_{2} \mathrm{SO}_{4}$ solution was used for electropolishing the surface at $6 \mathrm{~V}$ for 3 seconds. To image the surface of the sample, grain structure, a SEM equipped with a tungsten filament (model:Inspect S50, FEI, Hillsboro, OR, USA) and EDS (model: X-Max 20 Oxford Instruments, Concorde, MA, USA) were employed. In order to gain information on the surface roughness WLIM (model: NT3300, WYKO, Veeco Metrology, Tucson, AZ, USA) was used before and after the dissolution experiment.

After imaging the pristine nickel sample with the SEM and WLIM, the dissolution of the sample was investigated. The sample was dissolved using a potentiostat/galvanostat (model: PG590, HEKA, Germany) in a Watts electrolyte $\left[\mathrm{NiSO}_{4} \cdot 7 \mathrm{H}_{2} \mathrm{O}(240 \mathrm{~g} / \mathrm{L}), \mathrm{NiCl}_{2} \cdot 6 \mathrm{H}_{2} \mathrm{O}(20 \mathrm{~g} / \mathrm{L})\right.$, $\mathrm{H}_{3} \mathrm{BO}_{3}(20 \mathrm{~g} / \mathrm{L}]$, which is commonly used in industrial electroplating practices. The electrochemical conditions within the cell were selected to mimic industrial settings. The dissolution of the $\mathrm{CN} 1$ sample was completed at $60^{\circ} \mathrm{C}, \mathrm{pH}=4$, and a current density of 0.8 $\mathrm{A} / \mathrm{dm}^{2}$. The value of the current is a representative average current density used by electroplating 
companies. [7] The first two dissolutions were completed at 10 minute intervals (10 and 20 minutes total dissolution time), the next two dissolutions were completed at 20 minute intervals (40 and 60 minutes of total dissolution) and the final dissolution was recorded after a 60 minute interval (120 minutes total dissolution time). The samples were removed from the cell upon completion of each dissolution step, rinsed with Milli-Q water, and then dried for imaging. At the beginning and end of each dissolution interval, cyclic voltammetry was performed to understand the surface chemistry and to identify the potential ranges for the active, passive, and transpassive states.

The CN1 sample was imaged after each dissolution interval. SEM imaging was employed to understand surface texture and grain distribution. Imaging was performed using secondary electron detection (overall surface structure of sample) and backscattered electron detection (improved grain boundary contrast at high magnification). WLIM was used to obtain topographical imaging of the surface and to understand the surface roughness and periodicity of surface features. Scaling analysis was performed on each WLIM image to understand how the surface roughness changed over the period of dissolution, using the procedure described in [5-8].

To investigate the dynamics of pits formation, an in situ cell for optical microscopy was designed using a petri dish fitted with a glass coverslip (MatTek Corporation, MA, USA). A carbonyl nickel pellet (Vale Canada) working electrode was mounted in insulating epoxy and mechanically polished as described above. The polished sample was rinsed with Millipore water and allowed to sit in air $\sim 20$ hrs to allow the natural oxide to develop. No further electropolishing or electrochemical characterization was done on the sample to ensure that a pristine polished surface was imaged at $t=0 \mathrm{~s}$. The working electrode was mounted in the petri dish so that the separation between the working electrode (nickel pellet) and coverslip was approximately 
$260 \mu \mathrm{m}$. Nickel wire (99.98\% purity, $0.5 \mathrm{~mm}$, Alfa Aesar, Tewksbury, MA, USA) was embedded in the cell as a counter electrode with a circular geometry around the working electrode. An in situ nickel wire was also used as a reference electrode. Room temperature Watts electrolyte, of composition described above, was used as the supporting electrolyte. Constant current experiments were executed with a PAR Model 173 Potentiostat/Galvanostat (Princeton Applied Research, NJ, USA) in galvanostat mode at a current, which corresponded to $0.4 \mathrm{~A} / \mathrm{dm}^{2}$ based on the geometric area of the working electrode. Microscopy was performed on an AccuScope 3035 series inverted metallurgical microscope (Accu-Scope Inc., Commack, NY, USA) equipped with a 10 MP digital capture device and Micrometrics SE Premium Software (MicroMetrics, Ottawa, ON, CA). The software enables the auto-capture of time-lapse images throughout the experiment.

\section{Results and Discussion}

\subsection{Pristine Nickel Sample}
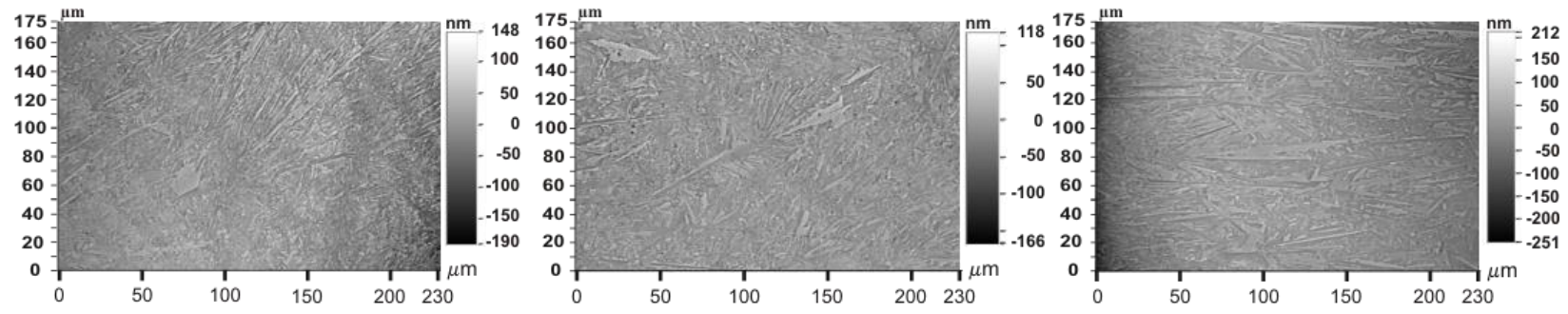

Left $\left(\sim 280^{\circ} \mathrm{C}-\sim 240^{\circ} \mathrm{C}\right)$

Right $\left(\sim 220^{\circ} \mathrm{C}-\sim 200^{\circ} \mathrm{C}\right)$
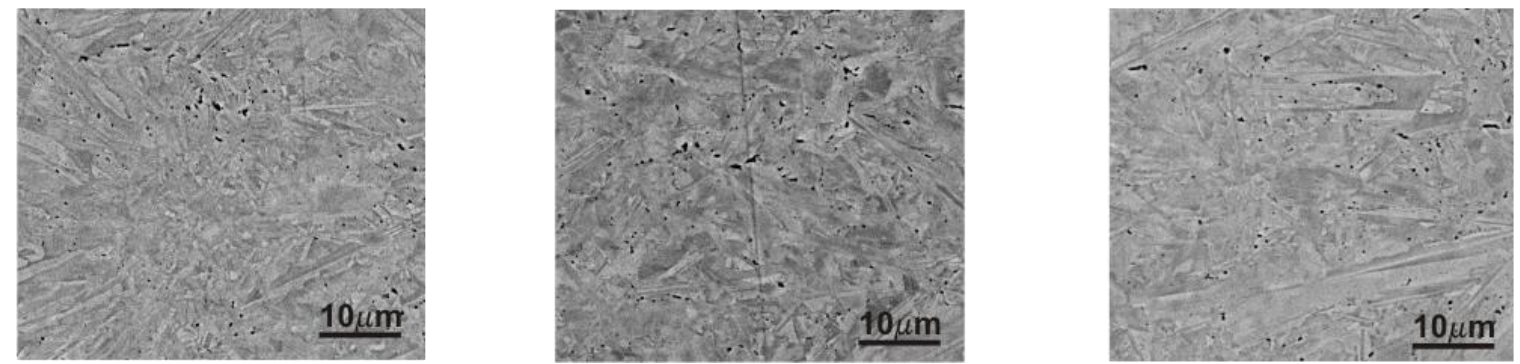
Figure 1: (a) WLIM Images of the CN1 sample - top row (b) Backscattered SEM Images of the CN1 sample -bottom row

The initial characterization of the sample surface was carried out by SEM and WLIM. Figure 1 shows the WLIM (top row) and backscattered SEM (bottom row) images of the CN1 pristine sample. The SEM images show the texture of the surface at a magnification that is approximately tenfold higher than the WLIM images. The decomposition temperature in this sample was decreasing from left to right. As the decomposition temperature is changed the grain size and structure are altered as well. The lamina deposited at $\sim 200^{\circ} \mathrm{C}$ has large grains consisting of large needle-like crystals. In the central region (grown at the decomposition temperature $\sim 240^{\circ} \mathrm{C}$ ), the size of grains is reduced and the number of smaller grains is increased. In addition, a slight increase in porosity of the surface could be seen. The needle-like crystals are still present in the left lamina (grown at $\sim 280^{\circ} \mathrm{C}$ ), however these crystals are much smaller and narrower than those observed on the right side of the sample (decomposition temperature $200^{\circ} \mathrm{C}$ ).

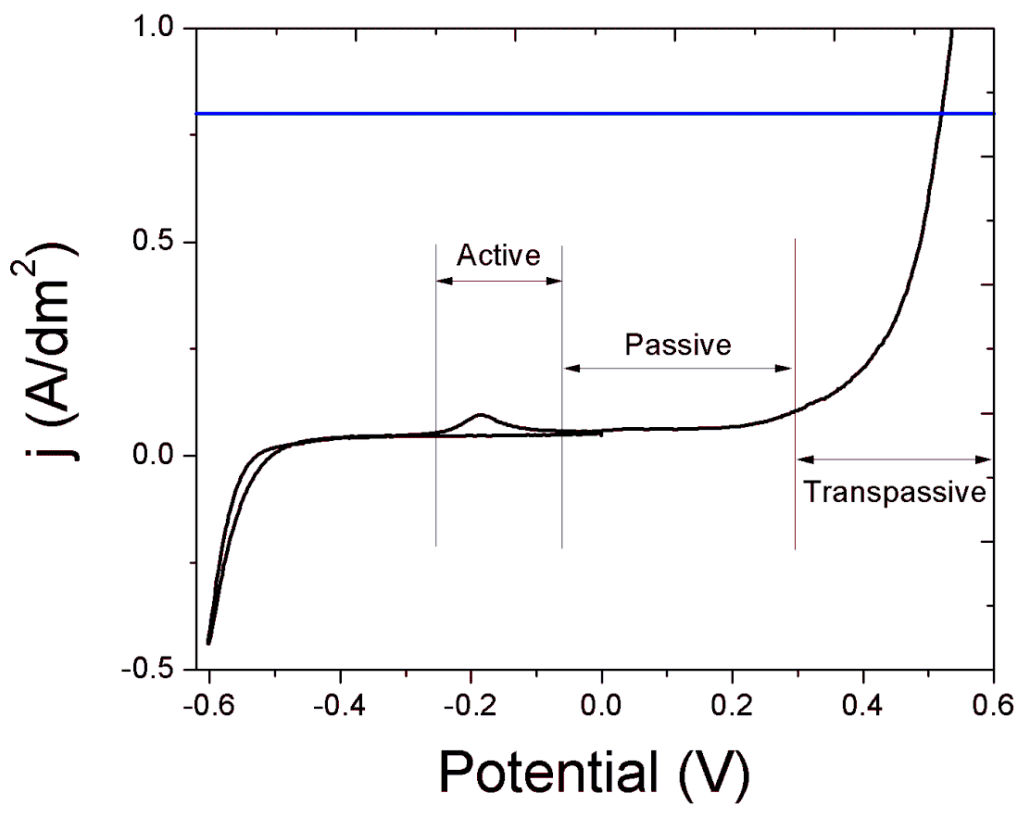

Figure 2: Cyclic voltammetry of the pristine CNI sample surface. The blue line indicates the current density applied during electrochemical dissolution. The CV was recorded in Watts Electrolyte at $60^{\circ} \mathrm{C}$ with a voltage sweep rate of 5 $\mathrm{mV} / \mathrm{s}$. A Ni sheet immersed in the Watts solution was used as the reference electrode. Potentials measured versus a Ni plate in the same electrolyte. 
Cyclic voltammetry (CV) was applied to determine the electrochemical behaviour of the sample. Figure 2 shows the $\mathrm{CV}$ curve recorded using a scan rate of $5 \mathrm{mV} / \mathrm{s}$. The initial potential for recording the voltammogram was set to $0 \mathrm{~V}$ vs Ni plate and scanned in the negative direction into hydrogen evolution. Upon the return sweep in the positive direction a small active region between -300 and $-70 \mathrm{mV}$ is observed, followed by a much larger passive region between -70 and $300 \mathrm{mV}$ with the onset of the transpassive region (pitting region) occurring around $300 \mathrm{mV}$. The solid blue line crossing horizontally at $0.8 \mathrm{~A} / \mathrm{dm}^{2}$ represents the current density that was applied throughout the galvanostatic dissolution experiments.

\subsection{Dissolution of the Nickel sample}

\subsubsection{Changes of the electrode potential with time}

After completing of the initial characterization, anodic dissolution of the CN1 sample was performed. The potential of the Ni electrode was monitored as a function of the dissolution time. The potential versus time plot is presented in Figure 3. After the initial potential spike to $\sim 0.7 \mathrm{~V}$, oscillations in the potential occur during the first 100 seconds of dissolution before the

potential drops to a relatively constant value $\sim 0.3 \mathrm{~V}$. The initial spike in potential implies a breakdown of the oxide film and oscillations represent its regrowth and repeated breakdown process. 


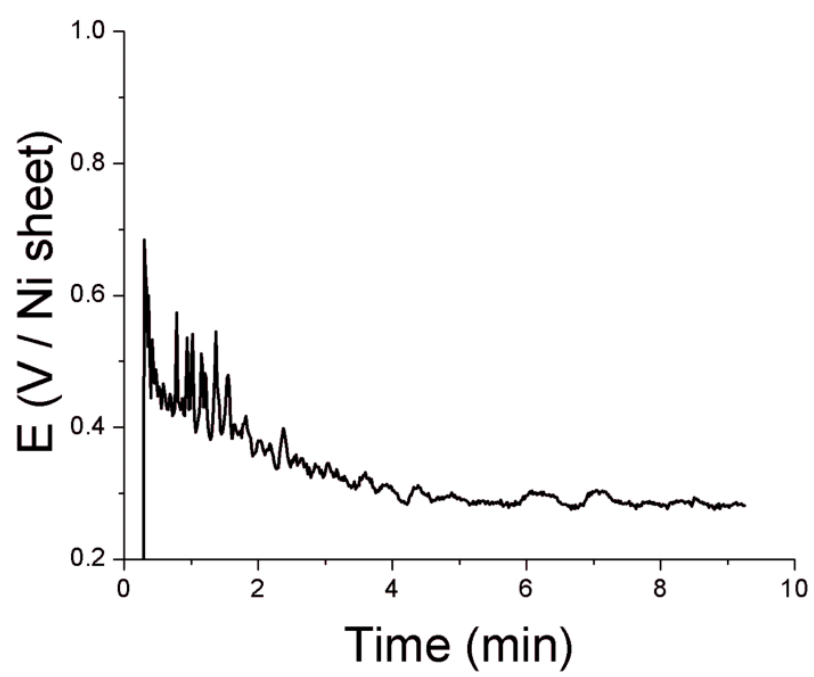

Figure 3: Potential vs Time plot showing the first 10 minutes of dissolution of the CN1 sample in Watts electrolyte at $60^{\circ} \mathrm{C}$ using a current density of $0.8 \mathrm{~A} / \mathrm{dm}^{2}$. The potential was measured against a Ni sheet placed in the same solution.

Once the value of the potential becomes constant at $\sim 0.3 \mathrm{~V}$, nickel dissolution is predominantly occurring at the end of the passive and beginning of the transpassive (pitting) regions (refer to Figure 2). It is well known that the breakdown of the passive film controls the initial Ni dissolution. [9-14] The passivity of nickel has been extensively studied by Marcus et al. $[11,13]$. It has been determined that the passive film is $0.9-2.5 \mathrm{~nm}$ thick and has a bilayer structure with an inner $\mathrm{NiO}$ layer and an outer hydrated nickel hydroxide layer $[11,13]$. Several papers discussed the breakdown of the passive layer. [13,15-25] In brief, the breakdown of the film may take place either at defect sites or by a large potential drop across the film causing thinning of the passive layer or electrostriction and formation of a micro-pit. [13,22] This step is followed by the penetration of anions to the metal surface through the micro-pit leading to the dissolution of the metal. The final step in metal dissolution involves the transport of the metal cations to the electrolyte solution. The presence of $\mathrm{Cl}^{-}$ions, at high enough concentrations, inhibits repassivation of the micro-pit assisting in passive film breakdown and dissolution of metal surfaces. [13,17] 
Chronopotentiometric curves similar to that shown in Figure 3 were recorded for samples during 20, 40 and 60 min dissolution. After inspection of the 60 minute dissolution using imaging techniques, the nickel sample was reintroduced to the electrochemical cell and further changes of the electrode potential with time were recorded. Figure 4a shows the chronopotentiometric curves that illustrate how the sample is dissolved at extended periods of time. The initial 30 min section of this plot is similar to the curve shown earlier in Figure 3. However, after about 40 min of dissolution significant oscillations began and continue till the end of this experiment. Similar oscillations have been observed in studies of the dissolution of stainless steel. [26-32] In corrosion literature they are sometimes referred to as noise. [33-37] Pistorius and Burstein describe the noise as originating from the nucleation, growth and repassivation of the microscopic pits. [33] Noise is a stochastic phenomenon. However, Figure $4 \mathrm{~b}$ illustrates that this noise has a regular periodicity of $\sim 0.8 \mathrm{~min}$ and quite regular amplitude of $\sim 0.5 \mathrm{~V}$ occurring between potentials corresponding to the end of the passive region at $\sim 0.3 \mathrm{~V}$ and the transpassive region $\sim 0.8 \mathrm{~V}$. These are characteristics of spatiotemporal phenomena, [26-32] which indicate that the surface is periodically repassivated and that extra potential was required to breakdown the repassivated layer to continue the dissolution process.

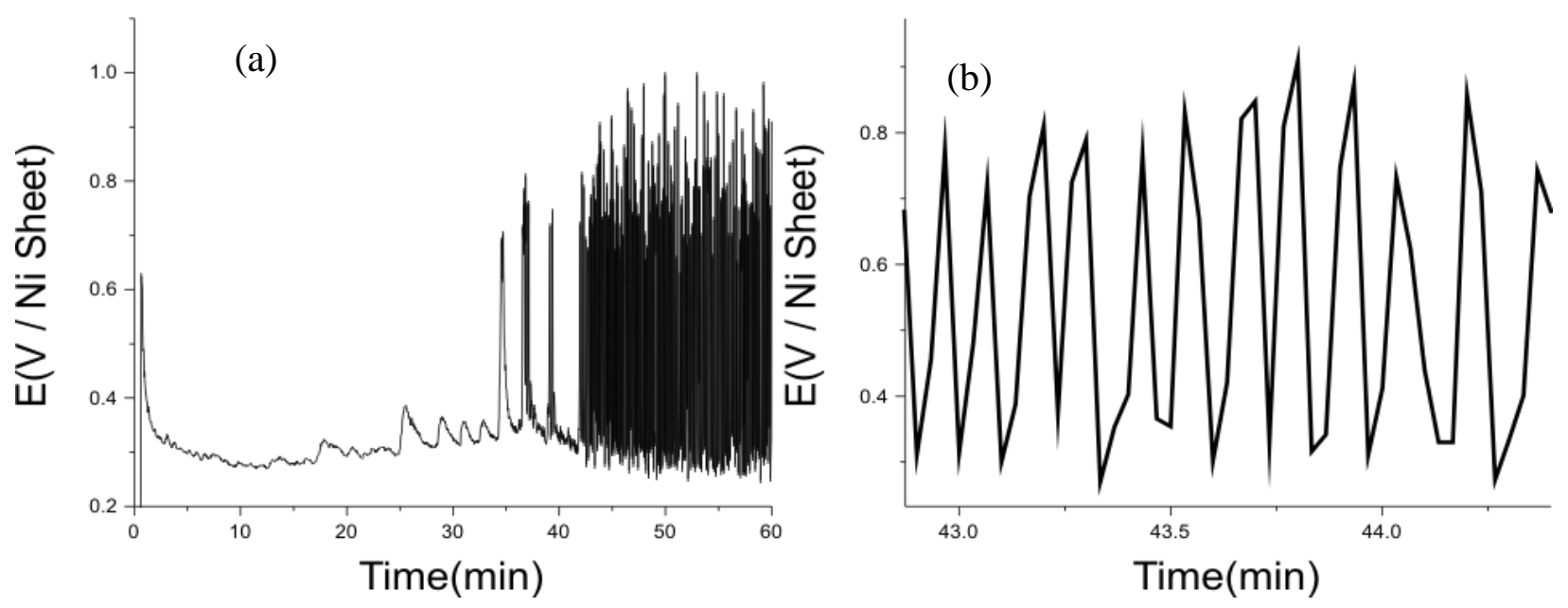


Figure 4: Chronopotentiometric curves recorded on electrode that was previously dissolved during 60 min, examined by SEM and WLIM and introduced into the electrochemical cell: (a) changes of potential during 60 min period additional dissolution and (b) zoomed in fragment showing the oscillations pattern.

In order to understand the changes to the surface morphology during the dissolution, optical microscopy, SEM and WLIM imaging were applied to observe these changes as a function of the dissolution time.

\subsubsection{Surface Characterization Studies of Temporal Nickel Dissolution}

\subsubsection{Initial Stages of the Sample Dissolution}

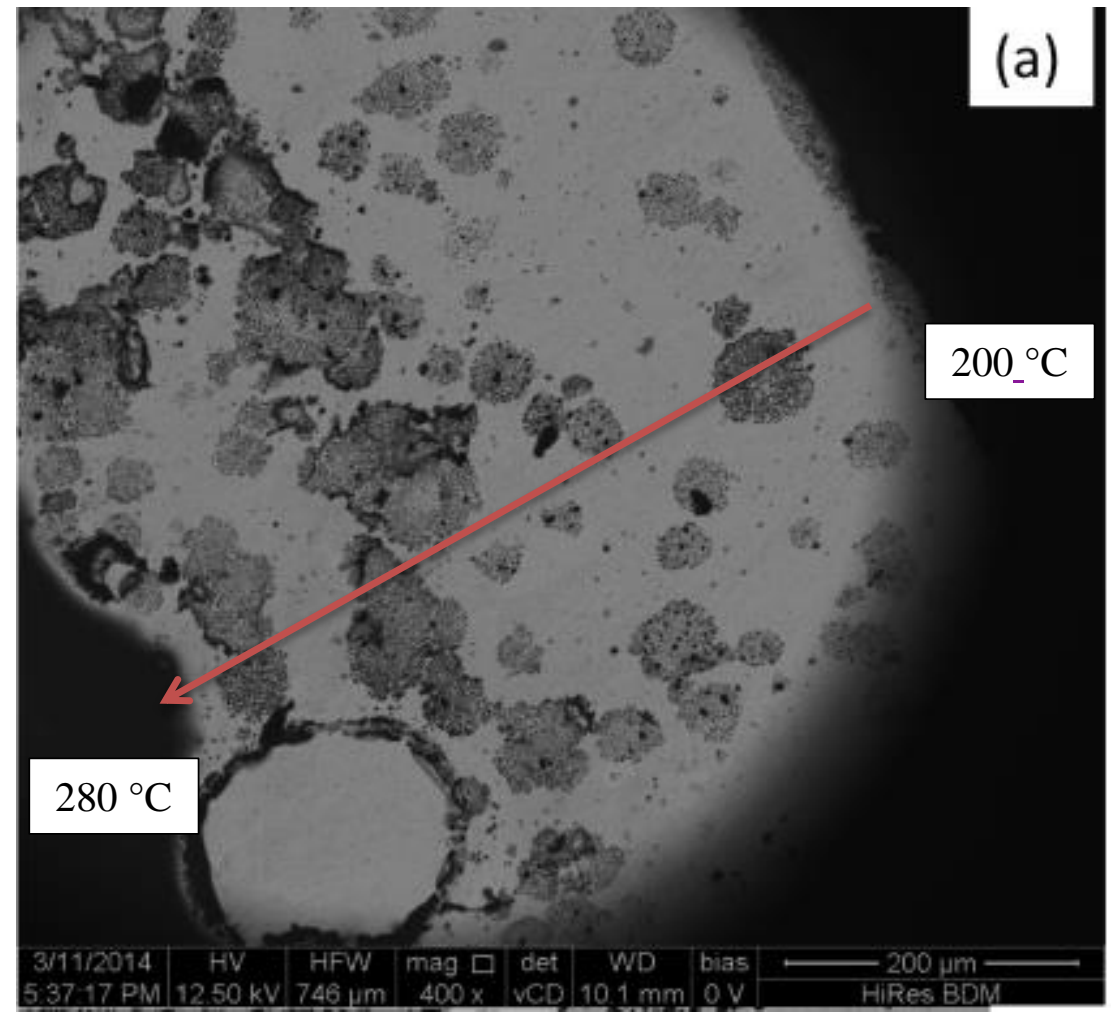

Figure 5: SEM image of the CN1 sample after 10 minutes of dissolution in the Watts electrolyte at $60^{\circ} \mathrm{C} \mathrm{and} \mathrm{i}=0.8 \mathrm{~A} / \mathrm{dm}^{2}$. The red arrow indicates the growth direction of the sample (right to left).

Figures 5 shows the SEM image of the CN1 sample after 10 minutes of dissolution. The majority of the grain structure seen in Figure 1 was removed during the dissolution process leaving a rough surface with multiple micropits. The arrow on the image represents the direction of the growth of the sample corresponding to changes of the decomposition temperature from 
200 and $280^{\circ} \mathrm{C}$. The image shows that the dissolution is more pronounced from the central to left portion of the sample corresponding to the Ni grown at decomposition temperatures between 240 and $280{ }^{\circ} \mathrm{C}$. The surface is covered by clusters of pits with lacy covers. The formation of pits with lacy covers has frequently been observed in corrosion research. [26-37] The growth of these pit clusters begins with a breakdown of the passive layer creating a single localized micropit $[26,33]$. In the presence of $\mathrm{Cl}^{-}$, the repassivation is inhibited allowing for further dissolution inside of the pit and increased flux of $\mathrm{Ni}^{2+}$ out of the pit. [38] The $\mathrm{H}_{3} \mathrm{O}^{+}$concentration also increases inside the pit leading to a localized acidification around the pit. The diffusion of $\mathrm{H}_{3} \mathrm{O}^{+}$ ions outward from the pit has a hemispherical geometry. Diffusing $\mathrm{H}_{3} \mathrm{O}^{+}$weakens the passive layer within the hemispherical region and initiates secondary pitting. [34-36] From this point, the process begins with the secondary pits further decreasing the $\mathrm{pH}$, which causes more $\mathrm{H}_{3} \mathrm{O}^{+}$ions to diffuse into the surrounding solution environment. This process results in an autocatalytic progression of circular pits clusters along the surface. Budiansky et al. [31,32] have observed this phenomenon in corrosion of stainless steel and other groups have also found that this mechanism is occurring during the anodic growth of $\mathrm{TiO}_{2}$ nanotubes $[39,40]$.

Figures 6a to c show zoomed images of pits with lacy covers observed in selected fragments of the sample following a 10-minute dissolution. 

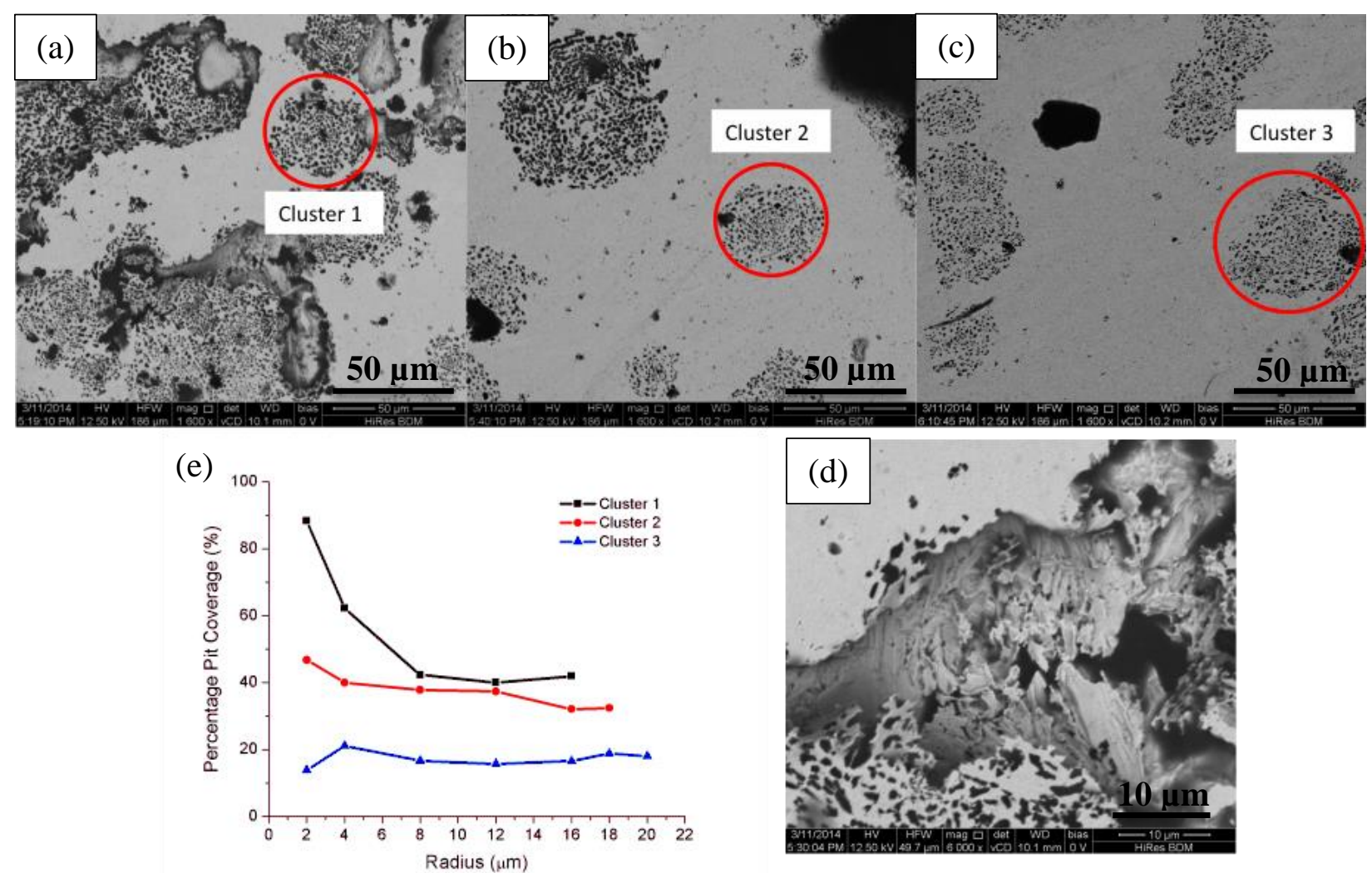

Figure 6: Pit cluster formations after 10 minutes of dissolution. (a) Cluster one from the left portion of the sample (decomposition temperature $\sim 260^{\circ} \mathrm{C}$ ) (b) the right side (decomposition temperature $\sim 200{ }^{\circ} \mathrm{C}$ ) and (c) middle fragment of the sample (decomposition temperature $\sim 240{ }^{\circ} \mathrm{C}$ (d) plot of the percentage of pits covering the cluster area at a given radius from the center of the cluster(e) partially collapsed lacy cover at the left side of the sample (decomposition temperature $260^{\circ} \mathrm{C}$ )

The micropit clusters show a circular distribution pattern. The areas surrounding the clusters appear to be relatively free of pits. This is most evident in the image present in Figure 6c, which was taken from the right side of the sample (decomposition temperature $200^{\circ} \mathrm{C}$ ). The image in Figure 6d, taken from the left side of the sample (decomposition temperature $\sim 260^{\circ} \mathrm{C}$ ) where more dissolution took place, shows that some of the lacy covers have collapsed forming open pits. For the three clusters of micropits marked by circles in Figures 6a-c, the radial density of micropits was calculated and plotted as a percentage of the area covered by pits in a disc with a radius $r$ drawn from the center of the cluster in Figure 6e. For cluster 1 (Figure 6a), the pit density is $\sim 90 \%$ at very short distance from the center of the pit cluster, but the pit coverage decreases and levels off to a value $\sim 43 \%$ at sufficiently larger distances. Clusters 2 (Figure 6b) 
and 3 (Figure 6c) are observed at the right and central segments of the sample (decomposition temperature $\sim 200^{\circ} \mathrm{C}$ - and $\sim 240^{\circ} \mathrm{C}$, respectively) where the dissolution has not progressed to the same extent. In these two cases, the distribution of pits within the cluster is more uniform. This behavior suggests an interaction between the localized pitting sites. The hemispherical diffusion geometry of aggressive ions emanating from the primary pit may contribute to the observed distribution of micropits. [31, 32]

Optical microscopy was employed to capture the stages of pit formation and propagation during Ni dissolution. This experiment was performed at room temperature $\left(20 \pm 2^{\circ} \mathrm{C}\right)$ in Watts electrolyte using a constant current density of $0.4 \mathrm{~A} / \mathrm{dm}^{2}$. The working electrode was mounted in a petri dish such that the gap thickness between the coverslip and working electrode was approximately $260 \mu \mathrm{m}$. [41] Figure 7 presents the images of the Ni surface at different times during the galvanostatic dissolution. Shortly after the application of the current, a primary central pit (marked by white arrow in Figure 7a) surrounded by concentric rings of perforations was observed, consistent with pit propagation under a lacelike cover as previously reported for corrosion in stainless steel. [26, 27, 34-37] The cover has a sunken appearance indicating sustained dissolution of $\mathrm{Ni}$ beneath the cover. For the first 9 minutes each optical image reveals new, quasi-circular perforation contours at increasingly larger distances from the pit centre (panel 7a). At 11 minutes (panel 7b) a new, discrete pit is observed (red arrows) at the periphery of the perforation boundaries and panel $7 \mathrm{c}$ reveals that the pit cover continues to expand. At 15 minutes (panel 7d) another secondary pit is formed (blue arrow) but subsequent images (panel 7e) indicate that the cover is no longer expanding indicating that the pit has repassivated. The perforation contour lines plotted in panel $7 \mathrm{f}$ reveal that the pit cover is initially quite circular but becomes increasingly elliptical with time. 


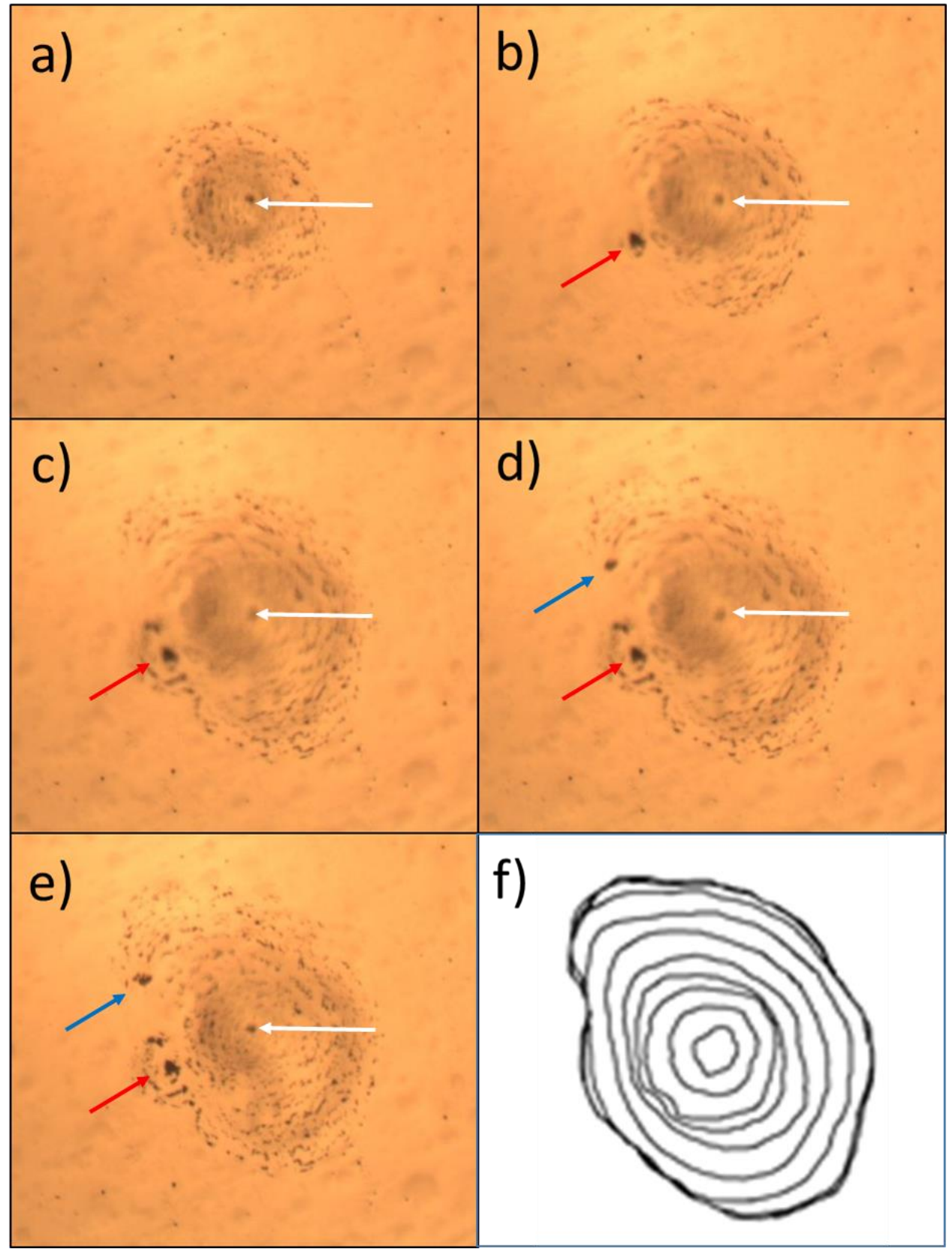

Figure 7: In situ optical microscopy images showing a radial pit cluster formation on a polished Ni pellet during

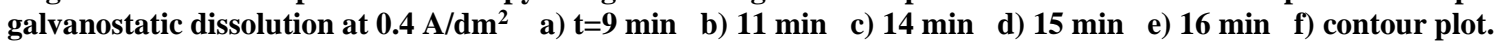


Figure 8 plots the major and minor pit cover radii as a function of dissolution time and the shape of the plots resemble two overlapping waves. The first wave shows a growth pattern which is closely proportional to $t^{1 / 2}$ and consistent with pit growth under diffusion control (Pistorius and Burstein [33]). By combining Faraday's law with Fick's first law the following relationship can be built for a pit propagating under hemispherical diffusion control [16]

$$
r^{2}=\frac{M D_{e f f} \Delta C}{\rho a} t
$$

where $r$ is the pit radius, $a$ is dimensionless constant related to the shape of the pit and equal to 1 for a hemispherical pit, $M$ and $\rho$ are the molar mass and density of the metal respectively, $D_{\text {eff }}$ is the effective diffusion coefficient that accounts for possible migration effects and $\Delta C$ is the concentration difference between the metal ion in the pit and the bulk solution. The inset in Figure 8 shows a plot of the average of $r^{2}$ versus the time for the first 8 minutes of pit growth and, using Eqn 2, the slope of the fitted linear line provides a value of $1.3 \times 10^{3} \mu \mathrm{m}^{2} \mathrm{~min}^{-1}$ and a value for $D_{e f f} \Delta C=3.2 \times 10^{-8} \mathrm{~mol} \mathrm{~cm}^{-1} \mathrm{~s}^{-1}$. 


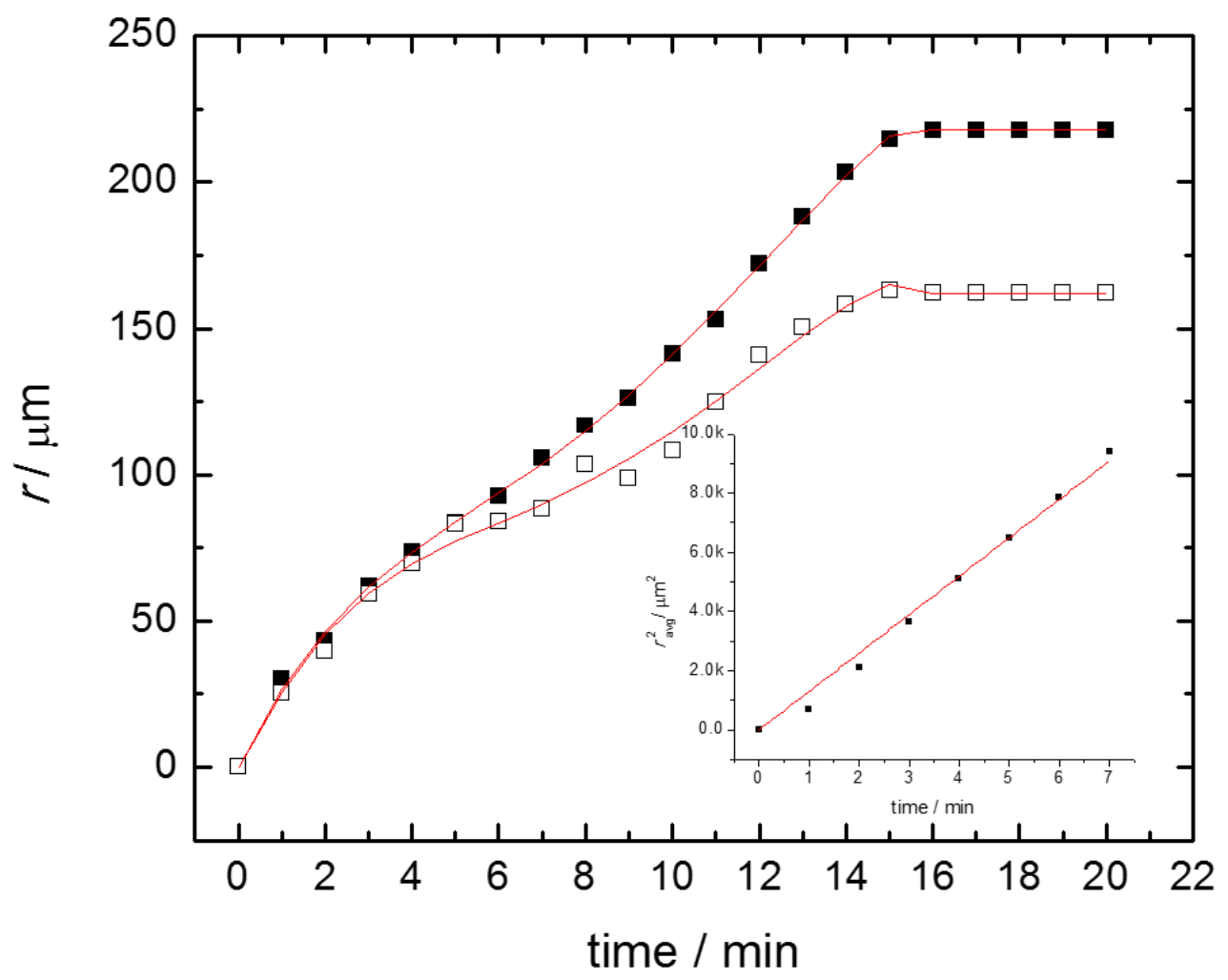

Figure 8: Dependence of the major and minor pit cover radii as a function of electrolysis time; inset plots the square of the average radius as a function of time for the first seven minutes of the pit growth.

It is illustrative to compare the measured value of $D_{e f f} \Delta C$ to predicted values. It is well established that metal dissolution is driven by aggressive species, in particular, chloride ions. The anolyte composition within the pit is determined by diffusion of anions into the pit and dissolved metal species out of the pit. Sustained pit propagation takes place when the concentration of metal ion (a surrogate measure of aggressive anion concentration) in the pit $\left(C_{\mathrm{M}}\right)$ corresponds to: $0.75 C_{\mathrm{s}} \leq C_{\mathrm{M}} \leq 1.5 \mathrm{C}_{\mathrm{s}}$ where $C_{\mathrm{s}}$ is the saturation concentration of the metal chloride [26,27,33,36,37]. At lower concentrations repassivation takes place whereas at concentration $1.5 C_{\mathrm{s}}$ the dissolution is slowed down by salt precipitation from the supersaturated solution of the 
anolyte. The factor 1.5 takes into account the fact that the anolyte is supersaturated when salt starts to precipitate [33]. In most corrosion studies the metal concentration outside the pit is equal to zero whereas in the present study the dissolution experiment is carried out in Watts electrolyte with a $\mathrm{Ni}^{2+}$ concentration of $\sim 1 \mathrm{M}$ and a mixture of sulfate and chloride ions. Sulfate ions are considered to be non-aggressive ions [16] and hence one can reach an upper limit estimate of pitsustaining anion concentration in the pit anolyte by assuming that only chloride ions enter the cavity to sustain ionic neutrality. At room temperature, the solubility of $\mathrm{NiCl}_{2}$ is $5.2 \mathrm{M}$. Therefore the minimum $\mathrm{NiCl}_{2}$ concentration within the pit needed to support sustained dissolution is $3.9 \mathrm{M}$. Under the limiting assumption that only chloride ions diffuse into the pit, the maximum value for the difference in the $\mathrm{NiCl}_{2}$ concentration inside and outside the pit, is $\Delta C_{\max } \approx 3 \mathrm{M}$. Using $1.0 \times$ $10^{-5} \mathrm{~cm}^{2} \mathrm{~s}^{-1}$ as the value for the diffusion coefficient of nickel the value of $D \Delta C_{\max }$ for an unobstructed pit is $3.0 \times 10^{-8} \mathrm{~mol} \mathrm{~cm}^{-1} \mathrm{~s}^{-1}$. The ratio $0 \leq D_{e f f} \Delta C / D \Delta C_{\max } \leq 1$ represents a measure of the perforation factor [37] with values approaching zero indicating a completely non-porous pit cover and values of unity equating to a completely open pit. The calculated perforation factor for the first 7 minutes of pit growth is nearly 100\%. It is surprising to see such a high perforation factor given that the pit grows under a lacy cover which would be expected to hinder the diffusion of corrosion products out of the pit. In fact, when the pit has a lacy cover, the diffusion of $\mathrm{Ni}^{2+}$ from a pit into the solution proceeds through a two dimensional array of holes. Such diffusion can be described by a model sketched in Figure S1 of the supporting information. Lindemann and Landsberg [42] provided a description of a nonlinear diffusion through an array of regular-spaced circular discs with open pits in the center (model Figure S1 of the Supporting Information). Lindemann and Landsberg have shown that the current density flowing through the open centers is equal to: 
$j=n F \Delta C D /\left(\delta+\left|\sum A_{n} \tanh \left(\frac{x_{n} \delta}{r_{2}}\right)\right|\right)$

where $\delta=(\pi D t)^{1 / 2}$ is the thickness of the diffusion layer, $\sum A_{n}$ is a function defined Scheller et al [43] and $x_{n}$ are zero values of the first order Bessel function of $r_{1}$-the radius of the pores- and $r_{2}$-is the half distance between pores in the lacy cover ( see Scheller et al [43] for the definition of $\sum A_{n}$, it includes Bellel functions of the zero and first order and $x_{n}$ are zero values of the first order Bessel function). The numerical values of $\sum A_{n}$ are tabulated by Scheller et al [43]. When the thickness of the diffusion layer $\delta$ is much larger than $\mathrm{r}_{2}$ then $\tanh \left(\frac{x_{n} \delta}{r_{2}}\right) \approx 1$ equation (2) simplifies to [42]:

$j=n F \Delta C D /\left(\delta+\left|\sum A_{n}\right|\right)$

Using $1.0 \times 10^{-5} \mathrm{~cm}^{2} \mathrm{~s}^{-1}$ for the $\mathrm{Ni}^{2+}$ diffusion coefficient, the values of $\delta$ are equal to $434 \mu \mathrm{m}$ for $1 \mathrm{~min}$ and $1734 \mu \mathrm{m}$ for $16 \mathrm{~min}$ of the present dissolution experiment. Using values of $r_{2} \approx 2 \mu \mathrm{m}$ taken as the average of half the distance between the pores in the lacy cover and calculating the porosity factor from the images as equal to $\left(\mathrm{r}_{1} / \mathrm{r}_{2}\right)^{2}=0.15 \pm 0.5$ one can show that the values of $\sum A_{n}$ in the denominator of equation 3 are negligible in comparison to the thickness of the diffusion layer (see Supporting Information). This estimate shows that under conditions of the present experiment the lacy cover is not impeding diffusion from and to the pit and hence the dissolution is consistent with models developed for diffusion from an open pit.

Figure $7 \mathrm{f}$ shows that after $\sim 15 \mathrm{~min}$ of dissolution the pit quenches and ceases to propagate in size. Pit quenching is often attributed to the loss of (partial) pit covers and an increase in the flux of corrosion products out of the pit. However, the optical images shown in Figure 7 do not demonstrate significant changes in the apparent porosity of the pit cover 
immediately prior to quenching. This further substantiates the argument above that the lacy pit cover does not restrict mass-transport. The quenching of the pit can be explained by considering that the experiments are performed under constant current conditions. With the assumption that the local activity of $\mathrm{Ni}$ corrosion remains constant during active pit growth, the local current density of the pit must decrease as the pit grows. By considering the polarization curve shown in Figure 2, the drop in pit current density must correlate with a drop in the pit potential. The growth rate is slowed by a decaying potential (ohmic control) until it is no longer sufficient to maintain the critical concentration and passivation occurs. The pit must transition from a transpassive state to a passive state. In other words, a pit growing under constant current dissolution must have a natural tendency to self-passivate as it grows.

\subsubsection{Nickel Dissolution at Extended Time Periods}

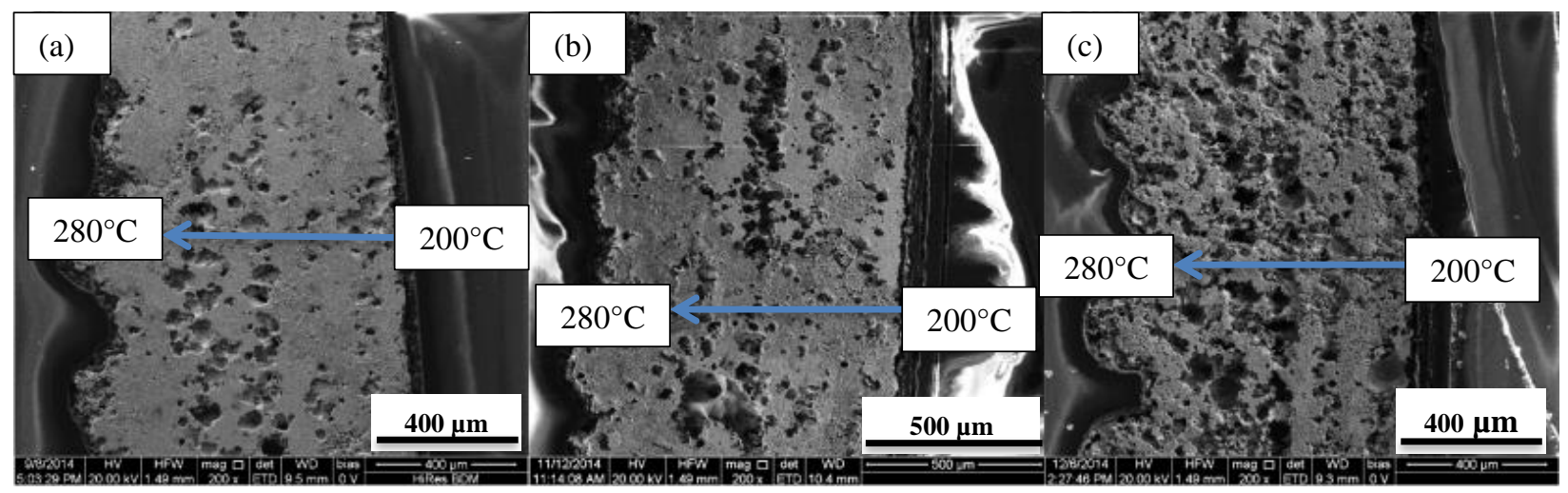

Figure 9: The SEM images of the CN1 sample after 40 (a), 60 (b), and 120 (c) minutes of dissolution in the Watts electrolyte at $60^{\circ} \mathrm{C}$ and $\mathrm{i}=0.8 \mathrm{~A} / \mathrm{dm}^{2}$. Arrows indicate direction of the sample growth.

The SEM images of the CN1 sample after 40, 60, and 120 minute dissolution are shown in $9 \mathrm{a}, \mathrm{b}$ and $\mathrm{c}$, respectively. The images show that with increasing dissolution time the surface becomes much rougher. The lamina formed at higher decomposition temperatures (left side of the sample) become significantly rougher and the pits in the left and central regions penetrate 
more deeply into the bulk of the sample than that of the right side of the sample (formed at lower decomposition temperature). The lacy covers that were present at the initial stage of the dissolution disappear and open pit formations dominate the latter dissolutions. Characteristically, the open pits form an array of stripes. One can distinguish four stripes of open pits that are separated by five smoother $\sim 200 \mu \mathrm{m}$ thick layers. This pattern corresponds well to the lamellar structure of $\mathrm{CN} 1$ sample and strongly suggests that the pitting and hence preferential dissolution takes place at the boundary between the five layers.

The SEM provides 2D images of the Ni surface with the third dimension available only for a visual inspection as a gray scale. In contrast, the WLIM allows one to acquire a digital 3D image of the Ni surface with numerical information about the height for every pixel of the imaged surface. WLIM was therefore employed to quantify the morphological information observed previously in SEM images. Figure 10 shows the topography of the CN1 sample after longer dissolution times. These images were constructed by stitching together several $90 \times 120$ $\mu \mathrm{m}$ images of the surface to obtain a greater representation of the surface topography while maintaining high resolution. The colour gradient for the height scale shows the height on the surface with respect to the average height. The positive and negative values indicate regions that

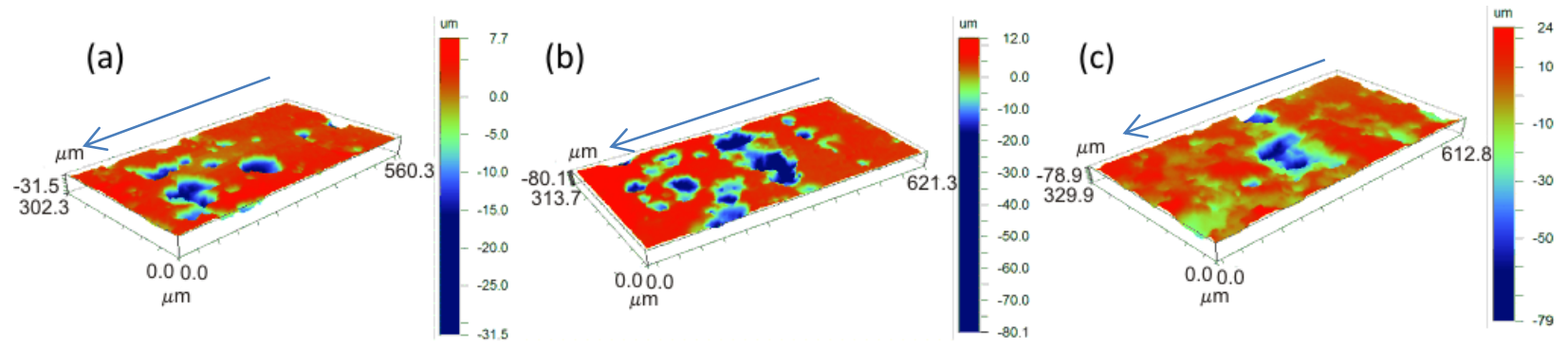

Figure 10: WLIM Images of the 40 (a), 60 (b), and 120 (c) minute dissolution. Arrow indicates direction of growth from $240^{\circ} \mathrm{C}-280^{\circ} \mathrm{C}$ at 40 minutes of dissolution, $220^{\circ} \mathrm{C}-260^{\circ} \mathrm{C}$ at 60 minutes of dissolution, and $240^{\circ} \mathrm{C}-280^{\circ} \mathrm{C}$ at 120 minutes of dissolution 
are either above or below the average height, respectively. The images show that the pits on the surface become deeper and the surface becomes rougher with increasing dissolution time.

These stitched images were taken from the central area of the sample. The SEM images in Figure 9 demonstrated that this region is the roughest area on the surface. Indeed, as the dissolution progresses the surface becomes extremely rough. Scaling analysis of the WLIM images presented in Figure 10 were used to quantify the surface roughness following the procedure described by Moula et al. [7] and the results have been summarized in Figure 11.

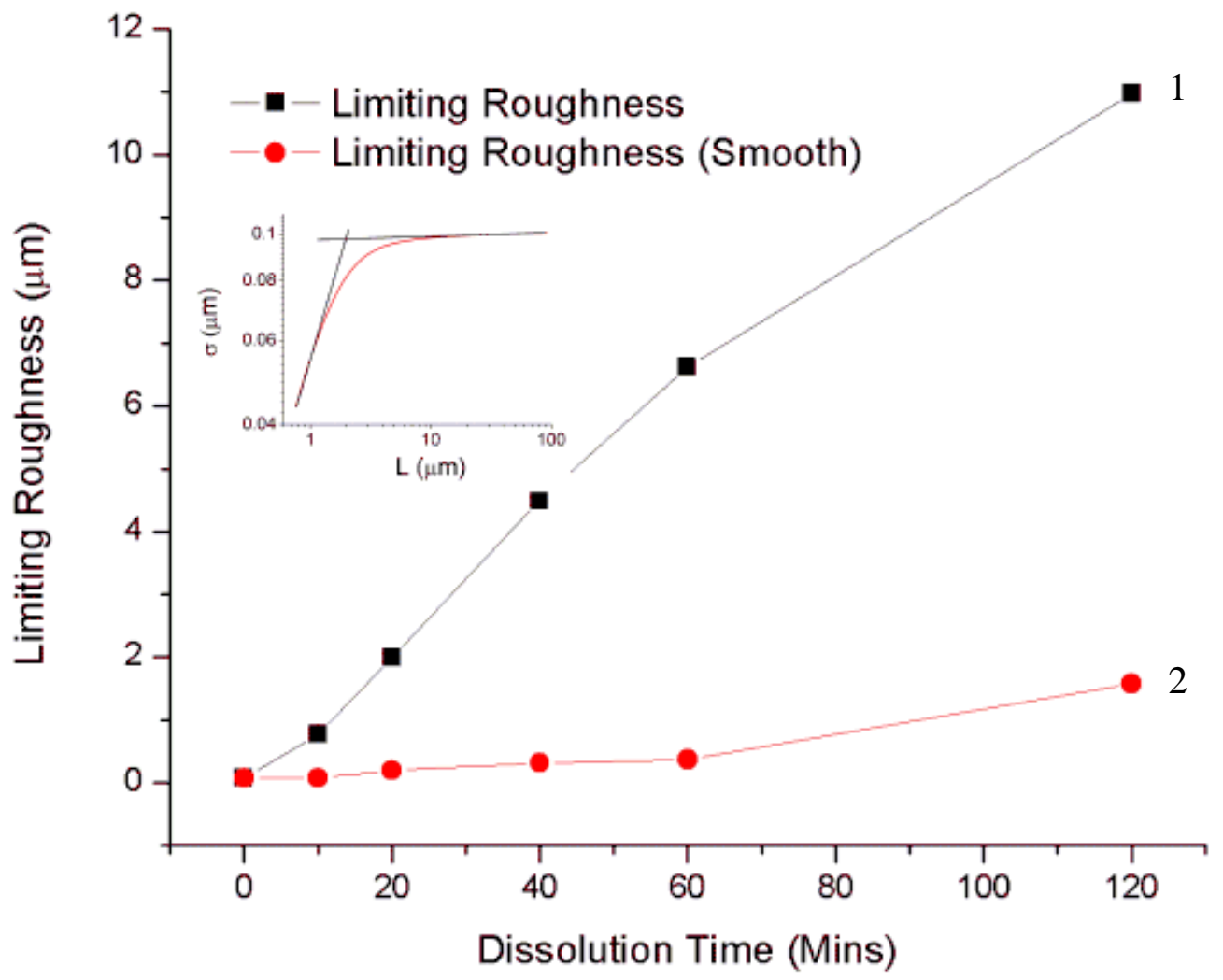

Figure 11: Surface Roughness Calculation completed using the WLIM images after each dissolution time. The black line (1) indicates full image surface roughness calculation. Red line (2) corresponds to the smooth (red) areas of the WLIM images (Figures 9 and 10). Inset shows scaling analysis curve (red), trend lines (black) intersect at point where the limiting roughness can be determined 
In scaling analysis [5-8], the root mean square (rms) of the surface height or the standard deviation of the surface heights is calculated within the square with a given length $L$. The square is then moved over the imaged surface by one-pixel increments in $\mathrm{X}$ and $\mathrm{Y}$-directions. The standard deviation of the surface height was calculated for each new position of the square. The standard deviations corresponding to all positions of the square were averaged to determine the average rms roughness of the surface $\left(\sigma_{L}\right)$ for a given length. At sufficiently large values of $L$, the parameter $\sigma_{L}$ attains a maximum value, which is called the limiting roughness. The peak-tovalley surface roughness is four-times that of the limiting rms roughness.

The black points (curve 1) in Figure 11 show the average limiting surface roughness plotted as a function of dissolution time. The roughness progresses almost linearly with the dissolution time. However, SEM and WLIM images show that pits contribute chiefly to the roughness of the surface and that the areas surrounding the pits are much smoother. Therefore, curve 2 and red points plot limiting surface roughness calculated for these "smooth" regions of the surface. The roughness of the "smooth" fragment also increases with time but these changes are about one order of magnitude smaller than the roughness calculated for the entire image. These results show quantitatively that the CN1 sample dissolves predominantly through pitting rather than in a layer by layer fashion. The pitting is observed along the boundary lines between laminas confirming that preferential dissolution of the laminar CN1 sample takes place at boundaries between laminas.

For narrow pits with a large aspect ratio, the WLIM instrument may have insufficient resolution to measure the pit depth. Therefore, additional SEM images were collected for cross sections of the sample after 120 min of the dissolution. Figure 12a shows the surface topography image of the sample while Figure $12 \mathrm{~b}$ shows the cross sectional image of this sample in the 
perpendicular direction. Figure 12a indicates that the profile of the cross section depends largely on the location of the cut. Figure $12 \mathrm{~b}$ shows that thickness of the corroded layer is smaller on the left side of the image corresponding to lower decomposition temperature and higher at the right side of the sample corresponding to higher decomposition temperature, consistent with previous experiments. For the low decomposition temperature the thickness of the corroded layer, estimated with the help of the SEM image, does not deviate too much from the peak to peak

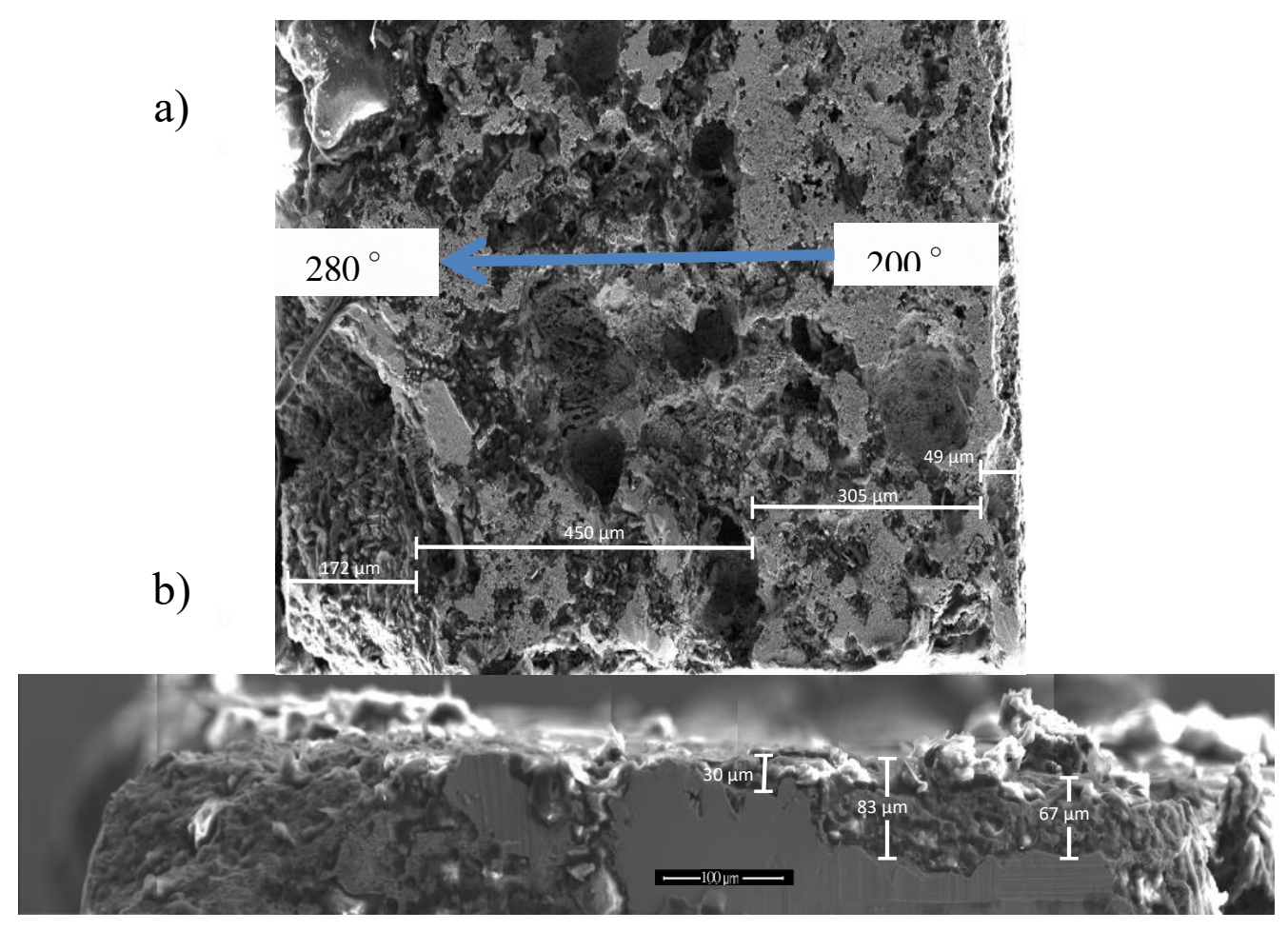

Figure 12: SEM images of the CN1 sample after 120 min dissolution: a) image of the surface topography of the cut fragment of the sample, b) image of the cross section the sample grown using decomposition temperature changing from 200 to $280^{\circ} \mathrm{C}$.

average roughness. The data in Figure 11 suggest that the average peak-to-peak roughness corresponds to about $45 \mu \mathrm{m}$. However, the corroded layer on the left side of the sample 
(produced at higher decomposition temperature) is thicker. In order to reveal the morphology of this layer, the sample was cut at the left side of the sample in the parallel direction. Figure 13 shows SEM image of the cross section of the lamina grown at $280^{\circ} \mathrm{C}$. Indeed, the thickness of this corroded layer is on the order of $\sim 100 \mu \mathrm{m}$, which is nearly double the peak-to-peak roughness measured by WLIM. Several rough saucer-shaped cross sections of pits that are $\sim 30-$ $40 \mu \mathrm{m}$ deep could also be seen in the SEM image. These pits were accessible to the WLIM and contributed to the rms roughness calculated from the WLIM images. They have rough surface in agreement with recent studies by Ghahari et al [37] who observed smooth pit formations in the dissolution of stainless steel using potentiostatic mode and rough pits in galvanostatic mode. However, the most striking information revealed by the image in Figure 13 is the presence of high density of micropits propagating deeply into the interior of the lamina. Effectively, the corroded layer has a

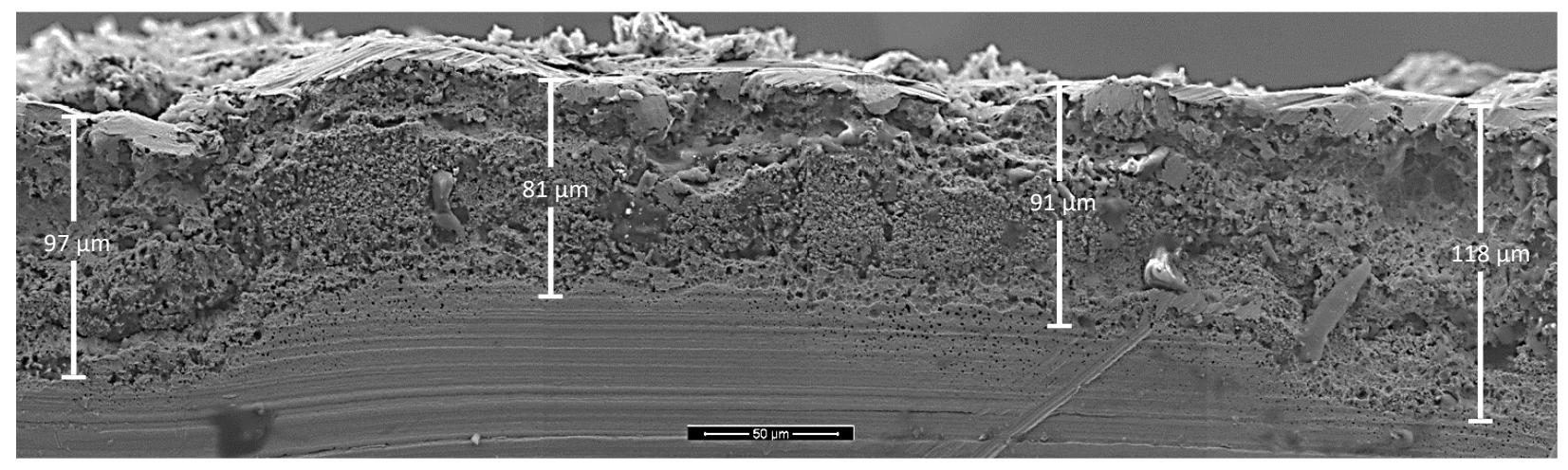

Figure 13: SEM image of the cross section of lamina grown at $\sim 280^{\circ} \mathrm{C}$ after $120 \mathrm{~min}$ of dissolution of the CN1 sample in the Watts electrolyte at $60^{\circ} \mathrm{C}$ and $\mathrm{i}=0.8 \mathrm{~A} / \mathrm{dm}^{2}$.

spongelike appearance. The uniformly dense Ni below the corroded layer indicates that porosity of that layer is a result of the dissolution and not the porosity of $\mathrm{Ni}$ in this lamina.

\section{Summary and Conclusion}


The mechanism of anodic dissolution of a laboratory made carbonyl Ni sample grown using the Mond process was investigated. The sample consisted of five $\sim 200 \mu \mathrm{m}$ thick lamina corresponding to $\mathrm{Ni}(\mathrm{CO})_{4}$ decomposition at temperatures ranging from 200 to $280^{\circ} \mathrm{C}$. At the initial stage of dissolution, pits with lacy covers were formed. With extended dissolution time, the pits with lacy covers were converted to open pits. The lamina grown at higher $\mathrm{Ni}(\mathrm{CO})_{4}$ decomposition temperature dissolved faster and its surface was rougher than laminae grown at lower decomposition temperatures. The most important observation in this study was formation of crevice lines along boundaries between laminas of $\mathrm{Ni}$ deposited at different temperatures. These crevice lines indicated that preferential dissolution of $\mathrm{Ni}$ occurs more rapidly along the boundary lines. A similar behavior was observed for dissolution of another Ni sample produced by carbonyl method using alternating temperatures and nickel carbonyl gas pressures. [44] The present study indicates that a variation in the decomposition temperature leads to a non-uniform dissolution of the sample. The knowledge provided by this study allows us to optimize $\mathrm{Ni}$ production by the carbonyl method for electroplating applications.

\section{Acknowledgments}

This work was supported by Vale Canada and the Natural Sciences and Engineering Research Council (NSERC) of Canada.

\section{REFERENCES}

[1] L. Mond, C. Langer, F. Quincke, L. - Action of carbon monoxide on nickel, J. Chem. Soc. Trans. 57 (1890) 749.

[2] L. Mond, The History of My Process of Nickel Extraction, J. Soc. Chem. Ind. 14 (11) (1895) 945-946. 
[3] C.M. Whittington, K.L.K. Yeung, W.Y. Lo, Refined nickel anodes: pointers to industrial best practice, Trans. IMF. 89 (3) (2011) 122-131.

[4] P.A. Moysey, N. V. Rama Rao, M.H.I. Baird, Dynamic coefficient of friction and granular drag force in dense particle flows: Experiments and DEM simulations, Powder Technol. 248 (2013) 54-67.

[5] D. Malevich, J.Y. Baron, G. Szymanski, J. Lipkowski, Optimization of the parameters for nickel electrowinning using interference microscopy and digital image analysis, J. Solid State Electrochem. 12 (4) (2008) 453-459.

[6] T. Zhao, D. Zagidulin, G. Szymanski, J. Lipkowski, Application of atomic force microscopy and scaling analysis of images to predict the effect of current density, temperature and leveling agent on the morphology of electrolytically produced copper, Electrochim. Acta. 51 (11) (2006) 2255-2260.

[7] M.G. Moula, G. Szymanski, B. Shobeir, H. Huang, I.J. Burgess, A. Chen, et al., Electrochemical Dissolution Behavior and the Residue Formation Mechanism of Laboratory Made Carbonyl Nickel, Electrochim. Acta. 162 (2015) 108-118.

[8] A.L. Barabasi, H.L. Stanley, Fractal Concepts in Surface Growth, in: Cambridge University Press, New York, 1995: p. 16.

[9] G.A. Di Bari, J. V. Petrocelli, The Effect of Composition and Structure on the Electrochemical Reactivity of Nickel, J. Electrochem. Soc. 112 (1) (1965) 99.

[10] M. Keddam, H. Takenouti, N. Yu, Transpassive Dissolution of Ni in Acidic Sulfate Media: A Kinetic Model, J. Electrochem. Soc. 132 (11) (1985) 2561.

[11] D. Zuili, V. Maurice, P. Marcus, Surface Structure of Nickel in Acid Solution Studied by In Situ Scanning Tunneling Microscopy, J. Electrochem. Soc. 147 (4) (2000) 1393.

[12] B. MacDougall, M. Cohen, Mechanism of the Anodic Oxidation of Nickel, $J$. Electrochem. Soc. 123 (12) (1976) 1783.

[13] P. Marcus, V. Maurice, H.-H. Strehblow, Localized corrosion (pitting): A model of passivity breakdown including the role of the oxide layer nanostructure, Corros. Sci. 50 (9) (2008) 2698-2704.

[14] E. Sikora, D.D. Macdonald, Nature of the passive film on nickel, Electrochim. Acta. 48 (1) (2002) 69-77.

[15] H. Boehni, Breakdown of passivity and localized corrosion processes, Langmuir. 3 (6) (1987) 924-930. 
[16] Strehblow, H-H.; Marcus, P. Mechanisms of Pitting Corrosion. In Corrosion Mechanisms in Theory and Practice; Marcus, P., Ed.; CRC Press, Boca Raton, USA, 2011; pp 349-393.

[17] G.S. Frankel, Pitting Corrosion of Metals: A Review of the Critical Factors, $J$. Electrochem. Soc. 145 (6) (1998) 2186-2198.

[18] J.R. Galvele, Transport Processes and the Mechanism of Pitting of Metals, J. Electrochem. Soc. 123 (4) (1976) 464-474.

[19] T.P. Hoar, D.C. Mears, G.P. Rothwell, The relationships between anodic passivity, brightening and pitting, Corros. Sci. 5 (4) (1965) 279-289.

[20] T.P. Hoar, The production and breakdown of the passivity of metals, Corros. Sci. 7 (6) (1967) 341-355.

[21] N. Sato, A theory for breakdown of anodic oxide films on metals, Electrochim. Acta. 16 (10) (1971) 1683-1692.

[22] N. Sato, K. Kudo, T. Noda, The anodic oxide film on iron in neutral solution, Electrochim. Acta. 16 (11) (1971) 1909-1921.

[23] J. Soltis, Passivity breakdown, pit initiation and propagation of pits in metallic materials Review, Corros. Sci. 90 (0) (2015) 5-22.

[24] D.D. Macdonald, Passivity-the key to our metals-based civilization, Pure Appl. Chem. 71 (6) (1999) 951-978.

[25] M. Wang, Potential Distribution, Shape Evolution, and Modeling of Pit Growth for Ni in Sulfuric Acid, J. Electrochem. Soc. 142 (9) (1995) 2986.

[26] P. Ernst, N.J. Laycock, M.H. Moayed, R.C. Newman, The mechanism of lacy cover formation in pitting, Corros. Sci. 39 (6) (1997) 1133-1136.

[27] N.J. Laycock, Perforated Covers for Propagating Pits, J. Electrochem. Soc. 145 (4) (1998) 1101.

[28] T.T. Lunt, Cooperative Stochastic Behavior in Localized Corrosion, J. Electrochem. Soc. 144 (5) (1997) 1620.

[29] B. Wu, Cooperative Stochastic Behavior in Localized Corrosion, J. Electrochem. Soc. 144 (5) (1997) 1614.

[30] L. Organ, J.R. Scully, A.S. Mikhailov, J.L. Hudson, A spatiotemporal model of interactions among metastable pits and the transition to pitting corrosion, Electrochim. Acta. 51 (2) (2005) 225 - 241. 
[31] N.D. Budiansky, J.L. Hudson, J.R. Scully, Origins of Persistent Interaction among Localized Corrosion Sites on Stainless Steel, J. Electrochem. Soc. 151 (4) (2004) B233.

[32] N.D. Budiansky, L. Organ, J.L. Hudson, J.R. Scully, Detection of Interactions among Localized Pitting Sites on Stainless Steel Using Spatial Statistics, J. Electrochem. Soc. 152 (4) (2005) B152.

[33] P.C. Pistorius, G.T. Burstein, Metastable pitting corrosion of stainless-steel and the transition to stability, Philos. Trans. R. Soc. Lond. Ser. A-Math. Phys. Eng. Sci. 341 (1992) 531-559

[34] M.H. Moayed, R.C. Newman, Evolution of current transients and morphology of metastable and stable pitting on stainless steel near the critical pitting temperature, Corros. Sci. 48 (2006) 1004-1018.

[35] P. Ernst, R.C. Newman, Pit growth studies in stainless steel foils. I. Introduction and pit growth kinetics, Corros. Sci. 44 (2002) 927-941.

[36] N.J. Laycock, S.P. White, J.S. Noh, P.T. Wilson, R.C. Newman, Perforated covers for propagating pits, J. Electrochem. Soc. 145 (1998) 1101-1108.

[37] M. Ghahari, D. Krouse, N. Laycock, T. Rayment, C. Padovani, M. Stampanoni, F. Marone, R. Mokso, A. J. Davenport, Synchrotron X-ray radiography studies of pitting corrosion of stainless steel: Extraction of pit propagation parameters, Corros. Sci. 100 (2015) 23-35

[38] J.N. Harb, R.C. Alkire, Transport and Reaction during Pitting Corrosion of Ni in 0.5M $\mathrm{NaCl}$, J. Electrochem. Soc. 138 (12) (1991) 3568.

[39] K. Huo, B. Gao, J. Fu, L. Zhao, P.K. Chu, Fabrication, modification, and biomedical applications of anodized TiO2 nanotube arrays, RSC Adv. 4 (33) (2014) 17300.

[40] P. Roy, S. Berger, P. Schmuki, TiO2 nanotubes: synthesis and applications, Angew. Chem. Int. Ed. Engl. 50 (13) (2011) 2904-39.

[41] I. Burgess, B. Barlow, G. Szymanski, J. Lipkowski, B. Shobeir, and B. Love, "Pulsed Potential Dissolution Reduces Anode Residue Formation During Nickel Electroplating", J.Electrochem.Soc., ,163 (2016) C164-170.

[42] J. Lindemann and R.Landsberg, J.Electroanal.Chem., Galvanostatische Einschaltmessungen an Teiweise Blokirten Modellelectroden, J.Electroanal.Chem., 29(1971)261-268.

[43] F.Scheller, S.Mueller,R.Landsberg,H-J.Spitzer, Gesetzmaessigkeit fuer den Diffusionsgrenzstrom an Teilweise Blockierten Modellelektroden, J.Electroanal.Chem.,19(1968) $187-198$ 
[44] A. Morrison, J.J. Leitch, G. Szymanski, B. Barlow, I. J .Burges, B. Shobeir, H. Huang, J. Lipkowski, Mechanism of Electrochemical Dissolution of Nickel Produced by the Carbonyl Method using Alternating Temperatures and Nickel Carbonyl Gas Pressures, Electrochim.Acta., submitted 